\title{
Spectroscopy, Circular Dichroism
}

National Cancer Institute

\section{Source}

National Cancer Institute. Spectroscopy, Circular Dichroism. NCI Thesaurus. Code

C19041.

Circular dichroism (CD), optical rotatory dispersion (ORD). Linearly polarized light can be thought of as a combination of right and left-handed circularly polarized light of equal phase and amplitude. ORD is the phenomenon observed when a substance exhibits a difference in the index of refraction of the right vs. left circularly polarized components, resulting in a change in the plane of polarization. When an optically active substance contains a chromophore that absorbs in the optically active region of the spectrum, CD is observed, which involves the preferential absorbance of right or left-handed circular polarized light. Both ORD and CD depend on the waveleng th of the radiation and the structure of the molecule. Structural elements of proteins that exhibit differences in $\mathrm{CD} / \mathrm{ORD}$ include the alpha helix, the beta sheet, and random coil segments. 